\title{
Telemonitoring of Capillary Blood Flow in the Human Skin: New Opportunities and Prospects
}

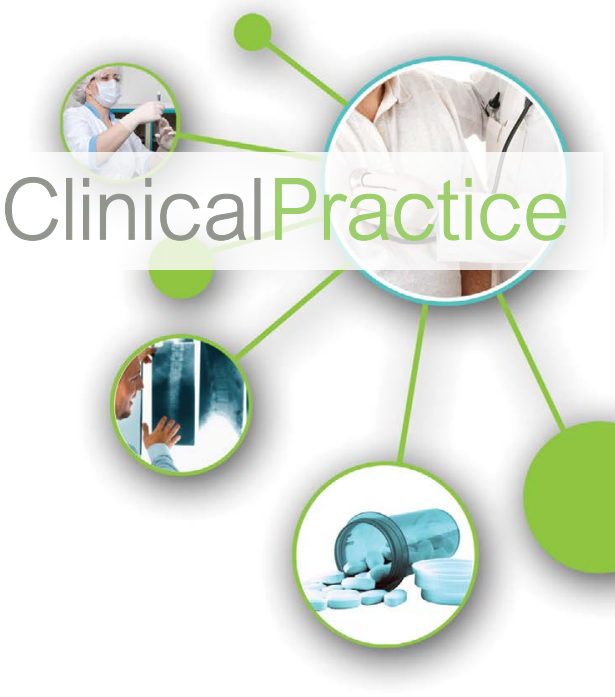

The oscillatory nature of cutaneous perfusion is fundamental and is caused by the functional activity of various regulatory mechanisms, which is confirmed by various methods of studying microcirculatory blood flow in humans. A conceptually new method for assessing skin microcirculation reviewed here is based on the analysis of video fragments of capillary blood flow in the skin registered with a conventional web camera (smartphone, tablet and others). The method makes it possible to evaluate the character of blood flow on the opposite to the heart "pole" of the greater (systemic) circulation - in the transitional part of the capillaries. Simplicity and usability of capillary blood flow telemonitoring in humans open way for a personalized approach to patient treatment as well as telemedical control of treatment effectiveness in a variety of pathological conditions. The following mini-review offers further insight into the subject.

\section{Keywords: microcirculation, vasomotions, capillaries, telemonitoring of capillary blood flow}

\section{Introduction}

The issue of studying and clinical evaluation of microcirculatory processes in humans is an important one not only for physiologists and pathophysiologists, but for clinicians as well. On the one hand, the parameters of microhemodynamics serve as a "window" to the world of fundamental physiological processes in tissues; while on the other hand, both the health and quality of human life depend on the proper functioning of the microcirculation system. Therefore, it is not surprising that nowadays the microcirculatory bloodstream (MCB), which is the first to be involved in various pathological processes, is attracting increasing interest of specialists in various fields of medicine and is often the main "target" for a wide variety of pharmaceuticals.

Throughout the twentieth century, noninvasive methods of studying microcirculation in humans have been constantly improving. In the last 2-3 decades, due to the rapid development of computer technologies and software, a number of promising study methods have appeared (computer capillaroscopy, laser Doppler flowmetry, laser speckle imaging, high-frequency ultrasound Doppler, photoplethysmography, infrared spectroscopy, thermal imaging, etc.), which allow non- invasive evaluation of structural and functional aspects of the human microcirculation system.

Due to their accessibility, the main objects for studying the MCB in humans are the oral mucosa, the eyes (the fundus and the bulbar conjunctiva), and the skin. Of all of these, the skin was the easiest to reach, which allowed measuring the blood flow velocity (A. Basler 1919) and the level of hydrostatic pressure (E. M. Landis 1930) in human capillaries already in the first third of the twentieth century [1,2]. A great advantage of the skin as an object for non-invasive studies of microcirculation in humans is that virtually all mechanisms of microcirculatory processes regulation (local, neurogenic sympathetic and nociceptive, humoral, etc.) are represented in it, and not only local but also most systemic processes as well are reflected in health and disease $[3,4]$. Another undeniable advantage of the skin is the ability to perform the widest range of various functional and pharmacological tests.

In order to interpret correctly the results obtained during microcirculation studies in the skin, it is necessary to have a clear idea of the object of the study, taking into account all its structural and functional features. The most detailed study of the structure and spatial organization of the skin MCB were performed
Fedorovich $A A^{1,2}$, Drapkina $\mathrm{OM}^{1}$, Pronko $\mathrm{KN}^{3}$, Sinopalnikov $\mathrm{VI}^{3}$ \& Zemskov $\mathrm{VM}^{* 3}$

${ }^{1}$ Federal State Institution, National Medical Research Center for Preventive Medicine of the Ministry of Healthcare of the Russian Federation, Moscow

${ }^{2}$ State Scientific Center of Russia, Institute of Biomedical Problems of the Russian Academy of Sciences, Moscow

${ }^{3}$ Department of Research and Development at Facecontrol, Inc., Florida, Miami, USA

*Author for correspondence: vzemskov@facecontrol.biz 
by Irwin Brawerman (1997). On the basis of a large array of biopsy material, using the technique of 1 micrometer $(\mu \mathrm{m})$ microtome sections, electron microscopy and computer modeling, the author showed that $1 \mathrm{~mm}^{3}$ of the papillary layer of the human skin in non-acral parts contains a typical microvascular module. The microvascular module comprises one feeding arteriole ascending from the depth of the dermis $(\leq 30 \mu \mathrm{m})$, five precapillary arterioles forming a network of capillaries that transform into postcapillary venules that merge into 9 collecting venules that flow into one descending draining venule $(\leq 50 \mu \mathrm{m})$. The author draws attention to the fact that structural organization of the MCB is similar in all preparations except for age differences in the number of exchange microvessels [5].

A feature of skin MCB is that the capillaries are located perpendicular to the skin surface. From the depth of the derma arterial part ascends, which at the skin surface makes a bend (transitional part), passing into the descending venous part of the capillary. The only visually accessible area of the skin capillaries along their entire length is the area of the nail bed. The skin over the nail root is thinned, stretched, and the capillaries are located parallel to the surface of the skin. This makes it possible, using capillaroscopy (CS), to evaluate their shape (FIGURE 1A), size, blood flow velocity, degree of interstitial space hydration [6], and other parameters.

When shifting as little as $0.5 \mathrm{~cm}$ to the interphalangeal joint (FIGURE 1B), the skin capillaries take a perpendicular position, and the CS shows only the transitional parts of the exchange vessels, i.e. the opposite to the heart "pole" of the greater circulation. A perpendicular arrangement of capillaries is true for all skin surfaces except for the skin in the nail bed area of the extremities.

Despite the highly specific and labor intensive nature of direct (invasive) measurement of capillary pressure in the human skin, published data from the 1920s to the present day contains more than 250 measurements in each of the capillary sections: arterial, transitional, and venous. The results show a difference in mean hydrostatic pressure from $32-38 \mathrm{~mm} \mathrm{Hg}$ in the arterial to $17-20 \mathrm{~mm}$ $\mathrm{Hg}$ in the transitional and $12-18 \mathrm{~mm} \mathrm{Hg}$ in the venous parts of the capillaries. Given the fact

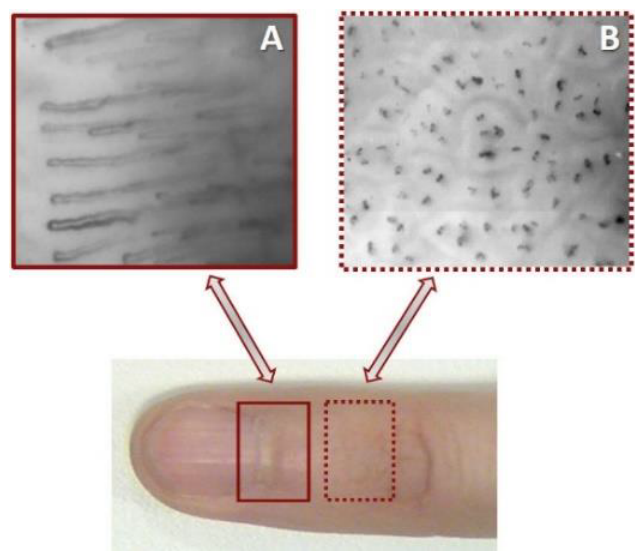

FIGURE 1. Capillaroscopy (CS). A - CS in the nail bed area. B - CS in the interphalangeal joint.

that the colloid osmotic pressure of the whole blood is normally virtually constant $(\approx 25 \mathrm{~mm}$ $\mathrm{Hg}$ ), the effective filtration and reabsorption pressure gradients in the arterial and venous parts of the skin capillaries are around 7-13 mm $\mathrm{Hg}$, respectively. This fully corresponds to the basic provisions of the Starling equation, which reflects the principles of action of the filtrationreabsorption mechanism of metabolism, which yields a consideration that the human skin MCB is a suitable and convenient object for the investigation of hemodynamic aspects of microcirculatory blood flow.

Studies have shown that pressure in the nail bed skin capillaries has a pulsating nature, and the shape of the capillary pulse wave is almost similar to the wave in the radial artery, and differs from the latter only by the oscillation amplitude. The speed of the pulse wave propagation from the radial artery to the nail bed capillary is about $10 \mathrm{~ms}$; along the capillary it averages $7.2 \mathrm{~cm} / \mathrm{s}^{-1}$ with a decrease in the amplitude from the arterial to the venous part of the capillary by approximately 30\% [7]. There are several characteristic forms of pressure changes. First of all, these are pulse oscillations with average pressure amplitude of 1-2 $\mathrm{mm} \mathrm{Hg}$. When the capillary sphincter relaxes, the amplitude of the pulse oscillations nearly doubles. The second form is irregular pressure changes lasting on average 15-20 seconds within 3-5 mm Hg. Pressure changes of the third type are more significant (up to 10 $\mathrm{mm} \mathrm{Hg}$ ) and long-lasting (5-8 minutes) [8]. As a result, pressure fluctuations from 14 to $71 \mathrm{~mm}$ $\mathrm{Hg}$ and from 11 to $52 \mathrm{~mm} \mathrm{Hg}$ are observed in the arterial and venous parts of skin capillaries, respectively [9]. Minimal changes in the pulse 

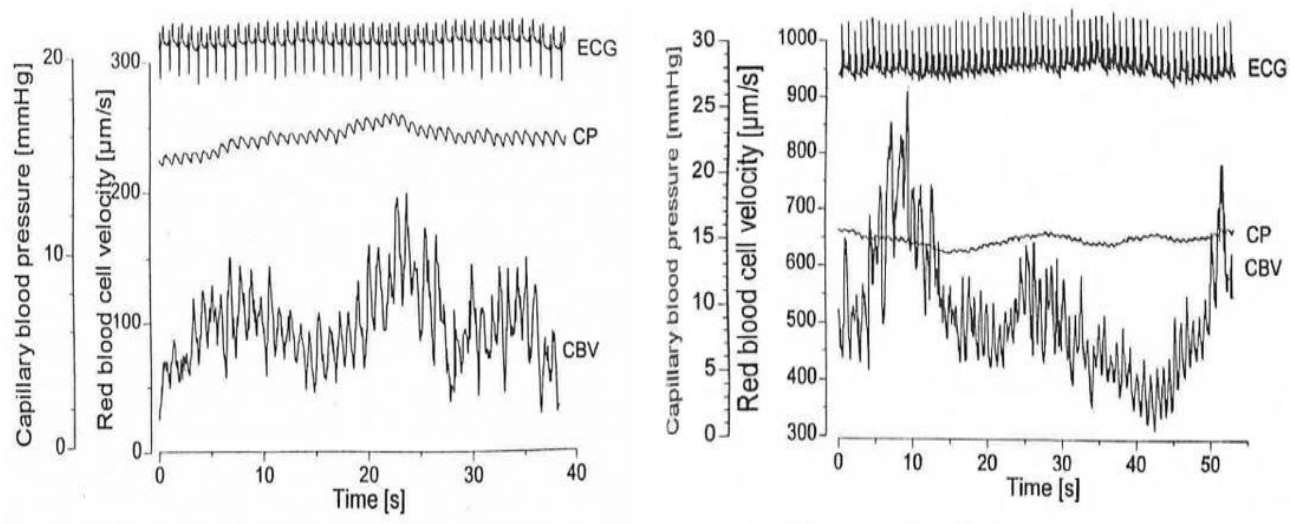

FIGURE 2. Simultaneous recording of ECG (ECG), pressure (CP) and blood flow velocity (CBV) in capillaries of the human nail bed. (Original figures from the article by Hahn M., Klyscz T., Jünger M. Synchronous measurements of blood pressure and red blood cell velocity in capillaries of human skin J. Invest Dermatol 1996 106: 1256-1259 Fig.3, p.1258; Fig. 5, p. 1259).

pressure lead to noticeable changes in the capillary blood flow velocity (CBV) with a high correlation $(\mathrm{R} 2=0.81)$ between the amplitude of pulse pressure fluctuations and the CBV [10]. The average CBV in the human skin capillaries in thermoneutral conditions is in the range of $400-900 \mu \mathrm{m} / \mathrm{s}$ and also has an oscillatory nature.

Using modern computerized capillaroscopes with high-speed digital video cameras and large magnification ( $\times 300$ and more) allows calculating CBV in various sections of a single capillary. However, to get correct results, it is necessary to analyze video fragments of maximum duration and with "perfect" (without "optical noise") image quality, which is extremely difficult to achieve in everyday clinical practice. The need for a long duration of recording capillary blood flow is due to its high variability (FIGURE 2), which is explained by the functioning of various regulatory mechanisms at the level of muscle microvessels, or precapillary arterioles.

A fundamental feature of precapillary arterioles is their high vasomotor activity. Arterioles are in constant motion, changing the tone and size of their lumen, which is manifested in the form of vasomotions ("vasa" - vessel, "motion" - movement) [11] with the respective changes in tissue perfusion (FIGURE 3-LDF). This is especially evident in laser Doppler flowmetry (LDF). Vasomotions of arterioles are brought about by the fact that smooth muscle cells of microvessels have their own basal tone and spontaneous contractile activity, contracting and relaxing on average 6 times per minute $(\approx 0.1 \mathrm{~Hz})$. It is the myogenic resistance at the level of the capillary sphincter that is the last link in the blood flow control just before the exchange vessels. Myogenic vasomotions are clearly passed into the capillary bed of the human skin [10,12], and their amplitude is positively interrelated with the number of functioning capillaries [13]. A large amplitude of myogenic vasomotions indicates a high degree of opening of capillary sphincters and precapillary arterioles, which facilitates the flow of blood directly into the capillaries. The tone and vasomotor activity of smooth muscle cells at the precapillary level are regulated by histomechanical, histometabolic, and oxygendependent mechanisms.

In more proximal segments of the arteriolar part of the MCB, the tone and vasomotor activity of the myocytes are modulated by neurogenic factors of various nature (sympathetic adrenergic and sensory peptidergic) with a frequency of $1.5-4$ oscillations per minute $(\approx 0.02-0.07 \mathrm{~Hz})$ from the external surface of the microvessel, and by endothelial factors less often than once a minute $(\approx 0.01 \mathrm{~Hz})$ from the internal lumen of arterioles. Each of the regulatory mechanisms operates in its frequency range, which can be evaluated by amplitude-frequency analysis (AFA) of blood flow oscillations [14]. Pulse oscillations amplitude reflects the influx of arterial blood to the MCB. The toneforming (endothelial, neurogenic, myogenic) mechanisms of arteriolar lumen regulation act through smooth muscle cells, modulating the volume of arterial blood flowing from large arterioles into the MCB to optimal values for the transcapillary exchange at a given site at and time. The higher the vasomotion amplitude of the tone-forming mechanisms, the greater the 
LDF

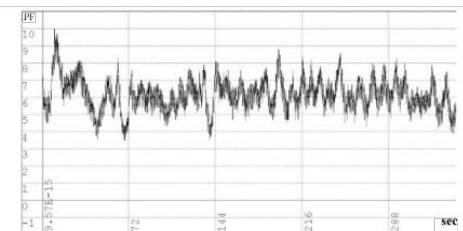

AFA

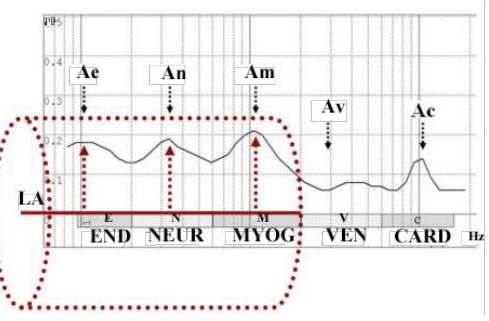

FIGURE 3. Laser Doppler flowmetry (LDF). LDF - the nature of tissue perfusion in $1 \mathrm{~mm} 3$ of the skin across six minutes. AFA - amplitude-frequency wavelet analysis of blood flow oscillations (logarithmic scale). Ac, Av, Am, An, Ae - is the amplitude of blood flow oscillations in the corresponding frequency range: cardial (card), venular (ven), myogenic (myog), neurogenic (neur), endothelial (end). LA is the longitudinal axis of the precapillary arteriole, the contours of which are marked by a dashed line (explanation in the text).

size of the precapillary arteriolar lumen and the lower the tone of this regulatory mechanism. And vice versa, the smaller is the amplitude of vasomotions, the smaller is the arteriolar lumen and the higher is the tone (FIGURE 3-AFA). The amplitude of respiratory-associated blood flow oscillations, in turn, reflects the state of the outflow pathways from the capillary bed (degree of venular plethora). During self-arrangement of microcirculation, all regulatory mechanisms interact with each other according to the principle of positive and negative feedbacks aimed at maintaining tissue homeostasis.

The oscillatory character of microcirculatory blood flow in the human skin is recorded not only in LDF and CS, but also in thermometry $[15,16]$, infrared spectroscopy [17,18], plethysmography $[19,20]$ and other study methods [21].

\section{Telemonitoring of capillary blood flow in the skin}

In 2017, the international team of scientists created a unique software product that allows assessing the capillary blood flow in any part of the human body based on the analysis of video fragments recorded from the skin surface using a standard webcam (computer, tablet, smartphone, etc.) (Application for the Eurasian patent for invention No. 201700326 dated 08.07.2017 "Telemetric system to monitor patient's vital functions"). The method is based on the analysis of changes in the contrast of pixels depending on the intensity of blood flow in the capillaries of the skin. Taking into account the angioarchitectonics of the skin MCB, the information obtained reflects the blood flow in the transitional parts of the capillaries, which are located directly at the skin surface (FIGURE 1B) and are the opposite to the heart "pole" of the greater circulation, where all metabolic processes occur.

FIGURE 4 shows an example of a threeminute video recording of cutaneous blood flow in the face area. The program automatically divides the study object into 4 sectors, which are marked with different colors. The total intensity of the original signal is reflected in the "Cr" sector. From the data presented, it can be seen that the level of the signal from the left half of the face (dark blue and blue) is slightly higher than in the right half (green and violet). In the "Filtered Cr" sector, a ten-second fragment is displayed in real time, with clearly visible pulse oscillations of the blood flow in the transitional part of the capillary. We see a cyclic change in the amplitude of pulse oscillations of the capillary blood flow, which is due to the vasomotor activity of precapillary arterioles and capillary sphincters. The higher the amplitude of pulse oscillations, the more arterial blood enters the capillaries, and the larger is the size of lumens of precapillary arterioles and capillary sphincters. The duration of pulse oscillations in the skin capillaries corresponds to the rhythmic activity of the heart (see sectors "Histogram RR" and "Scatterogram"). This allows evaluating various parameters of heart rate variability that correlate with the results of synchronous ECG recording.

When analyzing the video fragment throughout its entire length ( 3 minutes), we clearly see that the blood flow in the transitional parts of skin capillaries has an oscillatory nature (FIGURE 5), which is a very characteristic feature of microcirculatory blood flow in the skin. Even without the amplitude-frequency analysis (the software is in the final stage of development), but with the knowledge of the frequency of regulatory mechanisms functioning, we can definitely distinguish on the presented fragment the alternating endothelial (FIGURE 5A) and neurogenic (FIGURE 5B) vasomotions, which differ both in frequency and in amplitude. With a more careful analysis 


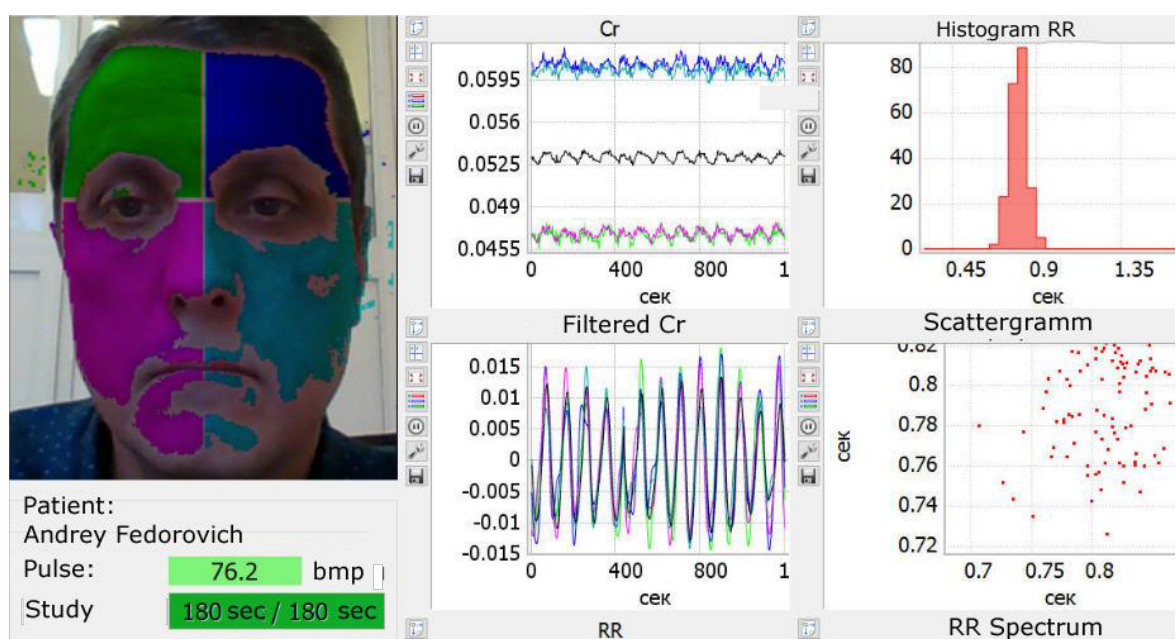

FIGURE 4. Telemonitoring of skin microcirculation in the facial area.

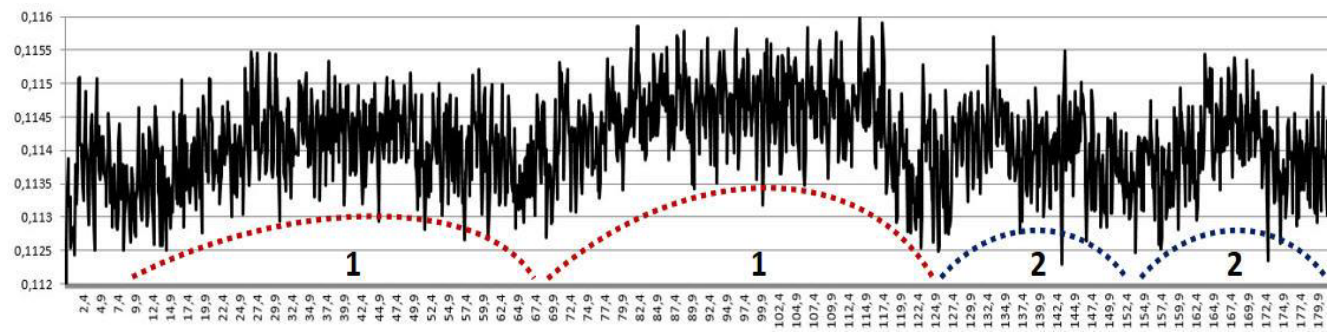

FIGURE 5. The nature of skin blood flow in the left half of the forehead (dark blue sector in Figure 4) for 180 seconds. Dotted lines: A - endothelial vasomotions; B - neurogenic vasomotions.

of the curve, it is possible to distinguish other mechanisms of microcirculatory blood flow modulation (myogenic, pulse and respirationassosiated), but more complete information on vasomotor activity of microvessels can be provided only by amplitude-frequency wavelet analysis.

Absolutely any skin surface area of the human body can be used for capillary blood flow telemonitoring, but it is the skin of the face that is of particular interest for several reasons. The first reason is regional features of skin microvessels' innervation, which is represented mainly by somatic sensitive (afferent) and vegetative sympathetic (efferent) regulation systems. Direct involvement of the parasympathetic nervous system in the regulation of cutaneous microcirculatory blood flow is considered proven for facial skin only $[22,23]$. Thus, the study of microcirculatory blood flow namely in the area of the facial skin opens the possibility for studying almost all (sympathetic, parasympathetic, sensory peptidergic) mechanisms of neurogenic control of resistive microvessels' vasomotor activity on the opposite to the heart "pole" of the greater circulation.
The second very important aspect is the anatomical features of the blood supply system. It is known that the head skin is fed from the external carotid artery system, and only the skin of the central part of the forehead is fed by supratrochlear (a.supratrochlearis) and partially by supraorbital (a.supraorbitalis) arteries, which are the terminal branches of the orbital arteries entering the system of the internal carotid arteries (FIGURE 6). The longstanding interest of researchers specifically to the ophthalmic artery basin is due to the fact that disorders of the microcirculatory blood flow in the eye area (the fundus and bulbar conjunctiva) are associated with various variants of cerebral circulation disorders [24-26]. In other words, the microcirculatory bed of the eyes is considered a "window" to the cerebral microcirculation system.

Our assumption is that analysis of microcirculatory blood flow in limited areas of the forehead skin, which are indicated by the dotted squares in FIGURE 6, will allow us to gain data on the state of cerebral blood flow in the basins of the right and left internal carotid arteries separately. As shown in the pilot study using the LDF method, the nature of the 


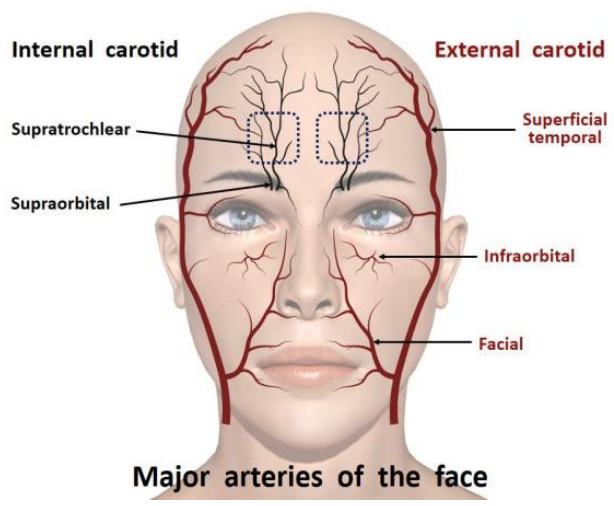

FIGURE 6. Anatomy of the blood supply to the skin of the facial part of the skull. Red color indicates the arteries from the external carotid artery (ECA) system, black color - the terminal branches of the orbital arteries entering the internal carotid artery (ICA) system. Dotted squares show areas of the skin for the analysis of the character of cerebral microcirculation in the basins of the right and left ICA.

microcirculatory blood flow in the forehead skin differs significantly both in the level of tissue perfusion and in the activity of the regulatory mechanisms of the microcirculatory blood flow depending on the side and size of cerebral vascular lesion. The authors also note significant regional differences in the nature of microcirculation during the first 3-7 days of cerebral blood flow recovery following thrombolytic therapy [27].

\section{Prospects}

The increased interest in the microcirculation system, which has been observed in the last few decades, can be explained by the emergence of a number of new instrumental methods for noninvasive examination of microcirculatory blood flow in humans. Studies are being actively performed in all leading scientific clinics on all continents and with the widest spectrum of abnormalities. Given that the microcirculatory bed vessels in most cases are the main "application point" of most pharmaceuticals, evaluation of the functional state of microvessels is a useful tool for analyzing the effectiveness of the therapy and, if necessary, its timely correction. All modern non-invasive methods for studying microcirculation are consistent with the principles of personalized medicine, but they require the patient's presence directly in the laboratory. That is the case when the patient comes to the doctor. And we know very well that most often, the patient comes to a specialist already at late stages of the disease, when various structural changes in the organs and tissues have been formed.

Due to its simplicity and usability, the method of telemonitoring capillary blood flow in the skin allows implementing the inverse case when the doctor comes to the patient. Given the wide-spread nature of various gadgets with web cameras, a doctor can come to almost every home. This approach, in addition to a wider coverage of the population, will allow the identification of diseases at an early stage of development (the stage of functional disorders), when the chances for full recovery are the highest, and the doctor possesses a wide array of various methods and means that facilitate the reverse process of the pathological process. In addition to compliance with the basic principles of personalized medicine, the method of telemetric control, by using Internet technology, allows sending finished reports and/or source video files for remote analysis, which meets the criteria imposed on telemedicine technology.

The possibility of evaluating vasomotor activity of resistive microvessels of the terminal branches of the orbital arteries entering the system of internal carotid arteries with the help of telemetric control is especially attractive. Information on the functional state of cerebral microvessels can expand significantly therapeutic possibilities in both acute (cerebral blood flow disorders, etc.), and in chronic (cerebrovascular insufficiency, Alzheimer's disease, etc.) pathological conditions. The results of assessing the functional state of cerebral microvessels at the time of headache attacks of various genesis (tension pains, migraines, $\mathrm{AD}$ differences, geomagnetic anomalies, etc.) may be very interesting as well. Another very useful application scenario is monitoring the physiological state of employees while at the workplace. This is especially relevant for activities that require increased attention or where the work conditions are unfavorable for humans. The scope of the method of capillary blood flow telemonitoring has no limits, but requires further research and development. 


\section{REFERENCES}

Basler A. Über die bestimmung der strömungsgeschwindigkeit in den blutkapilallen der menschlichen haut. Muench. Med. Wocheschr. 13, 347-348 (1919).

Landis EM. Micro-injection studies of capillary blood pressure in human skin. Heart. 15, 209-228 (1930).

Arck PC, Slominski A, Theoharides TC, Peters EM, Paus R. Neuroimmunology of stress: skin takes center stage. J. Investig. Dermatol. 126, 1697-1704 (2006).

Holovatz LA, Thompson-Torgerson CS, Kenney WL. The human cutaneous circulation as model of generalized microvascular function. J. Appl. Physiol. 105, 370-372 (2008).

Braverman IM. The cutaneous microcirculation: ultrastructure and microanatomical organization. Microcirculation. 4, 329-340 (1997).

Fedorovich AA. Non-invasive evaluation of vasomotor and metabolic functions of microvascular endothelium in human skin. Microvasc. Res. 84, 86-93 (2012).

Williams SA, Wasserman S, Rawlinson DW, et al. Dynamic measurement of human capillary blood pressure. Clin. Sci. (Lond.). 74, 507-512 (1988).

Zweifach BW. Quantitative studies of microcirculatory structure and function. II. Direct measurement of capillary pressure in splanchnic mesenteric vessels. Circ. Res. 34, 858-866 (1974).

Mahler F, Muheim MH, Intaglietta M, Bollunger A, Anliker M. Blood pressure fluctuations in human nailfold capillaries. Am. J. Physiol. 236, H888-H893 (1979).

Hahn M, Klyscz T, Jünger $M$. Synchronous measurements of capillary blood pressure and red blood cell velocity in capillaries of human skin. J. Invest. Dermatol. 106, 1256-1259 (1996).

Chambers R, Zweifach BW. Functional activity of blood capillary bed, with special reference to visceral tissue. Ann. NY Acad. Sci. 46, 683-694 (1944).

Meyer MF, Rose CJ, Hülsmann JO, Schatz H, Pfohl M. Impaired $0.1-\mathrm{Hz}$ vasomotion assessed by laser Doppler anemometry as an early index of peripheral sympathetic neuropathy in diabetes. Microvasc. Res. 65, 88-95 (2003).

Krupatkin AI, Sidorov VV, Fedorovich AA, Efimochkin SA, Zeinalov VT. The oscillatory circuit for the control of functional capillaries number. Reg. Hemodyn. Microcirc. 3, 54-58 (2006).

Krupatkin AI. Variations of blood flow - a new diagnostic language to the study of the microcirculation. Reg. Hemodyn. Microcirc.1, 83-99 (2014).

Podtaev S, Morozov M, Frick P. Wavelet-based correlations of skin temperature and blood flow oscillations. Cardiovasc. Engin. 8, 185-189 (2008).

Sagaidachnyi AA, Skripal AV, Fomin AV, Usanov DA. Restoration of the blood flow oscillations spectrum trough the finger temperature analysis and dispersion of the temperature signal in the biotissue. Reg. Hemodyn. Microcirc. 1, 76-82 (2013).

Zhang Z, Khatami R. Predominant endothelial vasomotor activity during human sleep: a near-infrared spectroscopy study. Eur. J. Neurosci. 40, 3396-3404 (2014).

Bosch BM, Bringard A, Ferretti G, Schwatz S, Idloi K. Effect of cerebral vasovotion during physical exercise on associative memory, a near-infrared spectroscopy study. Neurophotonics. 4, 041404 (2017).

Sun Y, Papin C, Azorin-Peris V, et al. Use of ambient light in remote photoplethysmographic systems: comparison between a hugh-performance camera and low-cost webcam. J. Biomed. Opt. 17, 037005 (2012).

Mizeva I, Di Maria C, Frick P, Podtaev $S$, Allen J. Quantifying the correlation between photoplethysmography and laser Doppler flowmetry microvascular lowfrequency oscillation. J. Biomed. Opt. 20, 037007 (2015).

Allen J, Howell K. Microvascular imaging: techniques and opportunities for clinical physiological measurements. Physiol. Meas. 35, R91-R141 (2014).

Drummond PD. The effect of sympathetic blockade on facial sweating and cutaneous vascular responses to painful stimulation of the eye. Brain 116, 233-241 (1993).

Izumi H. Nervous control of blood flow in orofacial region. Pharmacol. Ther. 81, 141-161 (1999).

Ikram MK, De Jonq FJ, Prins ND, et al. Retinal vessel diameters and cerebral small vessel disease: the Rotterdam Scan Study. Brain 129, 182-188 (2006).

Sharrett AR. A review of populationbased retinal studies of the microvascular contribution to cerebrovascular diseases. Ophthalmic Epidem. 14, 238-242 (2007).

Cheung N, Mosley T, Islam A, et al. Retinal microvascular abnormalities and subclinical magnetic resonance imaging brain infarct: a prospective study. Brain 133, 1987-1991 (2010).

Anisimova AV, Krupatkin AI, Sidorov VV, et al. Laser Doppler flowmetry in the assessment of the microcirculation in patients with acute and chronic cerebrovascular insufficiency. Reg. Hemodyn. Microcirc. 3, 31-37 (2014). 